\title{
Semiclassical approach to sequential fission in peripheral heavy-ion collisions
}

\author{
Andrea Strazzeri ${ }^{1}$ and Antonio Italiano ${ }^{2}$
}

${ }^{1}$ Dipartimento di Fisica e Astronomia dell'Università and INFN, Sezione di Catania, Catania, Italy

${ }^{2}$ INFN, Gruppo collegato di Messina, Messina, Italy

\begin{abstract}
A closed-form theoretical approach describing in a single picture both the evaporation component and the fast nonequilibrium component of the sequential fission of projectilelike fragments in a semiperipheral heavy-ion collision is derived and then applied to the dynamical fission observed in the ${ }^{124} \mathrm{Sn}+{ }^{64} \mathrm{Ni}$ semiperipheral collision at $35 \mathrm{~A}$ $\mathrm{MeV}$. Information on opposite polarization effects of the fissioning projectilelike fragments and on their "formation-to-fast fission lifetimes" are obtained.
\end{abstract}

\section{Introduction.}

In the past years several studies of sequential fission of the heavier product observed in peripheral heavy-ion collisions have revealed that the measured in-plane angular correlations between the fissioning projectilelike (PL) nucleus and the heavier fission fragment are sharply forward peaked and not symmetric with respect to the direction of the PL fragment with marked differences between distributions for positive and negative angles with a clear indication of a fast nonequilibrium component besides the equilibrated evaporative component $([1,2]$ and references therein). We here show that many of the features observed can be explained by means of a closed-form which combines in a single picture both the evaporation component and 
the fast nonequilibrium component of the sequential fission of the PL fragments following a peripheral heavy-ion collision. This approach is applied to the dynamical fission of PL fragments in the ${ }^{124} \mathrm{Sn}+{ }^{64} \mathrm{Ni}$ collision at $35 \mathrm{~A}$ $\mathrm{MeV}[2]$. Only an outline of the analytic expressions is given here; detailed calculations have been presented in an earlier brief paper [3].

\section{Theory.}

The theoretical background of our semiclassical approach is described in Ref. [4]. Firstly, let us consider a three-body sequential process such as $A+a \rightarrow B+b \rightarrow C+c+b$, where $C$ and $c$ indicate respectively the heavier and the lighter fragments from the fission of the heavier product of the peripheral collision $a(A, B) b$, the PL fragment $B$. From now on, $\vec{k}_{X}, \varepsilon_{X}^{*}, J_{X}$ and $m_{X}$ indicate the momentum, the excitation energy, the spin and its $z$-component, respectively, of the nucleus $X$. The spherical polar angles $\left(\vartheta_{B}, \varphi_{B}\right)$ of $\vec{k}_{B}$ are defined in the $(A+a)$ centre-of-mass (c.m.) system while $\vec{k}_{C}$ has polar angles $(\vartheta, \varphi)$ defined in the recoil-centre-of-mass (r.c.m.) system (rest frame of the nucleus $B$ ). We assume that the PL fragment $B$ is highly excited to levels that lie in the continuum region, within an energy interval $\Delta$ centered at the energy $\varepsilon_{B}^{*}$, and the fission fragments $C$ and $c$ are left in any of a large number of states contained within intervals $\Delta^{\prime}<\Delta$ about the energy $\varepsilon_{C}^{*}<\varepsilon_{B}^{*}$ and $\Delta^{\prime \prime}<\Delta$ about the energy $\varepsilon_{c}^{*}<\varepsilon_{B}^{*}$.

To get the average value of the $B-C$ angular correlation over the energy interval $\Delta$ centered at energy $\varepsilon_{B}^{*}$ let us split the $\mathcal{S}$ matrix, which describes the process $B \rightarrow C+c$, as $\mathcal{S}=\mathcal{S}_{E}+\mathcal{S}_{N E}$ with $\mathcal{S}_{N E} \equiv\langle\mathcal{S}\rangle$ and $\mathcal{S}_{E} \equiv(\mathcal{S}-\langle\mathcal{S}\rangle)[3,4]$ Then we can express the energy-averaged $B-C$ angular correlation as [3]

$$
\begin{gathered}
\left\langle d^{2} \sigma / d \omega_{B} d \omega\right\rangle=\left(d^{2} \sigma / d \omega_{B} d \omega\right)_{E}+\left(d^{2} \sigma / d \omega_{B} d \omega\right)_{N E} \\
\left(d^{2} \sigma / d \omega_{B} d \omega\right)_{E}=\sum_{m_{C}} \sum_{\ell} w_{\ell}\left\langle\left|S_{\ell}-\left\langle S_{\ell}\right\rangle\right|^{2}\right\rangle\left|\sum_{m_{B}} q_{\ell}\left(m_{B}, m_{C} ; \omega_{B}\right) Y_{\ell}^{m}(\omega)\right|^{2}
\end{gathered}
$$

where $m=m_{B}-m_{C}$, the amplitude $q_{\ell}$ determines the population of the substates $\left(J_{B} m_{B}\right)$ in the PL fragment $B$ and the quantity $w_{\ell}$ describes the probability of orbital momentum $\ell$ transferred in the decay $\left(B ; \varepsilon_{B}^{*} J_{B}\right) \rightarrow$ $\left(C c ; \varepsilon_{C}^{*} \varepsilon_{c}^{*} J_{C} \ell\right)$.

$$
\left(d^{2} \sigma / d \omega_{B} d \omega\right)_{N E}=\sum_{m_{C}}\left|\sum_{\ell}\left\langle S_{\ell}\right\rangle \sum_{m_{B}} q_{\ell}\left(m_{B}, m_{C} ; \omega_{B}\right) Y_{\ell}^{m}(\omega)\right|^{2},
$$


We choose coordinate axes having the $z$-axis along $\vec{k}_{B} \times \vec{k}_{A}$ (normal to the reaction plane) and the $x$-axis along $\vec{k}_{B}$, so that the direction $\varphi=0$ corresponds to the fragment $C$ moving along the PL fragment flight direction, and we suppose that the emission of the fragment $C$ does not change the sign of initial polarization of $\vec{J}_{B}$ at the moment of the fission of $B$.

Besides we consider $J_{B}$ and $J_{C}$ large as compared to $\ell$ so that the Clebsch-Gordan coefficient in Eq.(4) can be approximated by a reduced rotation matrix $d_{m \kappa}^{\ell}(\zeta)$ with $\kappa=J_{B}-J_{C}$ and $\cos \zeta=m_{B} / J_{B}$ [5].

We consider a coordinate rotation to a body-fixed system where the new quantization axis is oriented along $\overrightarrow{J_{B}}$ at a certain angle $\zeta$ with respect to the $z$-axis and lies in a plane perpendicular to the reaction plane and to the unit vector $\hat{k}_{0}$, oriented along the average momentum transferred in the collision $a(A, B) b$, corresponding to the angle $\varphi_{0}$ with respect to the $x$-axis [3]. Then, if we define as $(\Theta, \Phi)$ the polar angles of $\vec{k}_{C}$ with respect to the body-fixed $\left(\hat{k}_{0}, \hat{J}_{B} \times \hat{k}_{0}, \hat{J}_{B}\right)$ system, we have, for $\vartheta=90^{\circ}[6]$

$$
\cos \Theta=-\sin \zeta \sin \left(\varphi-\varphi_{0}\right), \quad \cot \Phi=\cos \zeta \cot \left(\varphi-\varphi_{0}\right) .
$$

Moreover, in Eq. (2) we get the parametrization $\left\langle\left|\left(\mathcal{S}_{E}\right)_{\ell}\right|^{2}\right\rangle=\left(T_{\ell} / G\right)$, with $T_{\ell}$ the optical model transmission coefficient and $G$ all decay modes energetically open for the decay $B \rightarrow C+c$, and in Eq.(3) we assume that the fast decay described by $\langle\mathcal{S}\rangle$ can be associated with a situation where the fragment $C$ is promptly emitted from a peripheral region of the nucleus $B$.

For the differential multiplicity, in units $\left(s r^{-1}\right)$, from Eqs.(1)-(4) we get [3]

$$
\begin{gathered}
M(\vartheta, \varphi, \zeta)=[M(\vartheta, \varphi, \zeta)]_{E}+[M(\vartheta, \varphi, \zeta)]_{N E}, \\
{[M(\vartheta, \varphi, \zeta)]_{E} \sim \sum_{\ell, \kappa} w_{\ell}\left(T_{\ell} / G\right)\left(Y_{\ell}^{\kappa}(\Theta, 0)\right)^{2},} \\
{[M(\vartheta, \varphi, \zeta)]_{N E} \sim\left|\sum_{\ell}\left\langle S_{\ell}\right\rangle_{+} \exp (+i \ell \Phi)\right|^{2}+H_{0}\left|\sum_{\ell}\left\langle S_{\ell}\right\rangle_{-} \exp (-i \ell \Phi)\right|^{2} .}
\end{gathered}
$$

In Eq.(7) $\left\langle S_{\ell}\right\rangle_{+}$and $\left\langle S_{\ell}\right\rangle_{-}$describe the splitting of the PL fragment with $m_{B} \equiv+m_{0}$ (positive polarization of $B$ ) and $m_{B} \equiv-m_{0}$ (negative polarization of $B$ ), respectively, and $H_{0}$ depends on the ratio between negative and positive polarization of the nucleus $B$.

The final expression of the differential multiplicity is given by $\left(\cos \zeta_{0}=\right.$ $\left.m_{0} / J_{B}\right)$

$M(\vartheta, \varphi)=\left(\int d \zeta \exp \left(-\left(\zeta-\zeta_{0}\right)^{2} / 2 \xi_{0}^{2}\right)\right)^{-1} \int d \zeta \exp \left(-\left(\zeta-\zeta_{0}\right)^{2} / 2 \xi_{0}^{2}\right) M(\vartheta, \varphi, \zeta)$, 
Here, for $M_{E}$ we get the semiclassical approach of Ref. [7]

$$
[M(\vartheta, \varphi, \zeta)]_{E}=C_{E} \exp \left(\gamma \sin ^{2} \Theta\right),
$$

where $C_{E}$ is a constant independent of $\vartheta$ and $\varphi$, and $\gamma$ is the so-called anisotropy coefficient.

If we admit than only a relatively narrow $\ell$-window centered at a certain $\ell_{0}$ contributes to the fast process described by $\langle\mathcal{S}\rangle$, for $M_{N E}$ we obtain [3]

$$
[M(\vartheta, \varphi, \zeta)]_{N E}=C_{N E}\left[\exp \left(-\left(\Phi+\chi_{0}\right)^{2} / \Gamma_{+}^{2}\right)+H_{0} \exp \left(-\left(\Phi-\chi_{0}\right)^{2} / \Gamma_{-}^{2}\right)\right],
$$

where $C_{N E}$ is a constant independent of $\vartheta$ and $\varphi, \chi_{0}$ is the quantal deflection and $\Gamma$ is the total angular dispersion. In the hypothesis that the PL fragment $B$ during the "formation-to-fast fission lifetime" $\tau_{0}$ rotates with rotational frequency $\omega_{0}=\hbar \ell_{0} / \mathcal{I}$ ( $\mathcal{I}$ is the moment of inertia) we assume [3]

$$
\chi_{0}=\omega_{0} \tau_{0}=\left(\hbar \ell_{0} / \mathcal{I}\right) \tau_{0} .
$$

Table 1: List of the parameters obtained in the analysis of the experimental in-plane angular correlations shown in Fig. 1 by the total multiplicity formula for three ranges of the total kinetic energy of the two heaviest fragments $E_{2 F}$, in units $M e V$, and for three heavy-light mass ratios $\left(A_{H} / A_{L}\right)$.

\begin{tabular}{cccccccccccc}
\hline \hline$E_{2 F}$ & $\left(A_{H} / A_{L}\right)$ & $C_{E}^{a}$ & $C_{N E}$ & $\gamma^{a}$ & $\zeta_{0}^{a}$ & $\xi_{0}^{a}$ & $\varphi_{0}^{a}$ & $H_{0}$ & $\Gamma_{+}$ & $\Gamma_{-}$ & $\chi_{0}$ \\
\hline \multirow{3}{*}{$3200-3650$} & $2.6-4.6$ & 0.3 & 4.8 & 3.0 & $6^{\circ}$ & $36^{\circ}$ & $16^{\circ}$ & 0.20 & 0.62 & 0.11 & $15^{\circ}$ \\
& $1.6-2.6$ & 0.9 & 3.8 & 2.9 & $6^{\circ}$ & $45^{\circ}$ & $10^{\circ}$ & 0.52 & 0.30 & 0.14 & $18^{\circ}$ \\
& $1.0-1.6$ & 1.2 & 3.0 & 2.8 & $6^{\circ}$ & $39^{\circ}$ & $8^{\circ}$ & 0.54 & 0.21 & 0.18 & $21^{\circ}$ \\
\hline \multirow{3}{*}{$2850-3200$} & $2.6-4.6$ & 0.3 & 6.0 & 3.0 & $6^{\circ}$ & $36^{\circ}$ & $23^{\circ}$ & 0.28 & 0.56 & 0.14 & $13^{\circ}$ \\
& $1.6-2.6$ & 0.7 & 3.6 & 2.9 & $6^{\circ}$ & $45^{\circ}$ & $20^{\circ}$ & 0.48 & 0.56 & 0.20 & $17^{\circ}$ \\
& $1.0-1.6$ & 1.2 & 2.4 & 2.8 & $6^{\circ}$ & $39^{\circ}$ & $17^{\circ}$ & 0.58 & 0.16 & 0.22 & $19^{\circ}$ \\
\hline \multirow{3}{*}{$2500-2850$} & $2.6-4.6$ & 0.2 & 5.6 & 2.9 & $6^{\circ}$ & $36^{\circ}$ & $22^{\circ}$ & 0.30 & 0.58 & 0.16 & $10^{\circ}$ \\
& $1.6-2.6$ & 0.6 & 4.4 & 2.7 & $6^{\circ}$ & $45^{\circ}$ & $20^{\circ}$ & 0.48 & 0.56 & 0.16 & $12^{\circ}$ \\
& $1.0-1.6$ & 1.6 & 1.6 & 2.6 & $6^{\circ}$ & $39^{\circ}$ & $18^{\circ}$ & 0.62 & 0.18 & 0.70 & $14^{\circ}$ \\
\hline \hline
\end{tabular}

${ }^{a}$ Quantities obtained by fitting the in-plane data in the region $|\varphi| \geq 100^{\circ}$ by the evaporative component.

\section{Application.}

Now, we briefly quote the application of the semiclassical approach above derived to the in-plane angular distributions of the heavier fragment from 


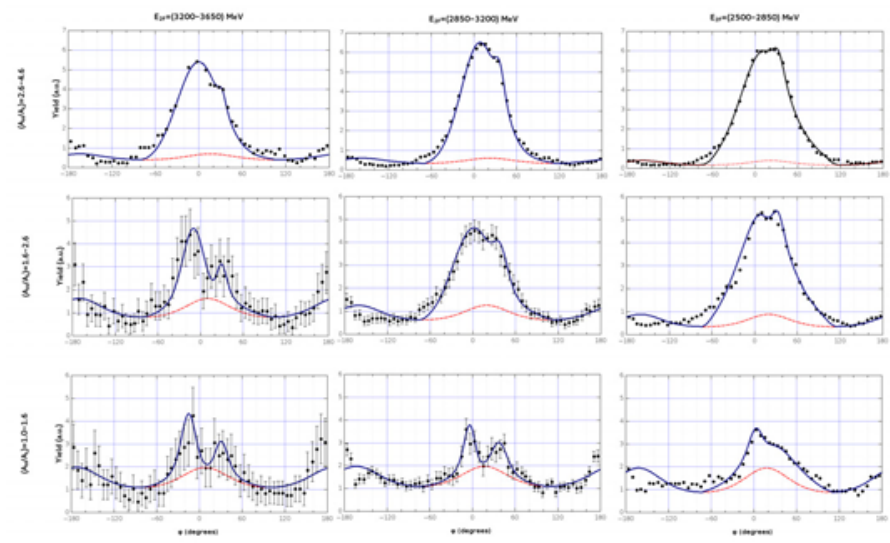

Figure 1: (Color online) In-plane angular distributions of the heavier fragment from the sequential fission of the projectilelike nucleus in the ${ }^{124} \operatorname{Sn}(35 \mathrm{~A} \mathrm{MeV})+{ }^{64} \mathrm{Ni}$ semiperipheral collision for three ranges of the total kinetic energy of the two heaviest fragments $E_{2 F}$, and for three heavy-light mass ratios $\left(A_{H} / A_{L}\right)$ [2]. The dashed and solid lines correspond to the equilibrium $M_{E}$ and the total $M_{E}+M_{N E}$ multiplicities, respectively (see text).

the fission of the PL nucleus in the ${ }^{124} S n+{ }^{64} N i$ semiperipheral collision at $35 \mathrm{~A} \mathrm{MeV}$ reported in Fig. 5 of Ref. [2].

The parameters $\left(C_{E}, \gamma, \zeta_{0}, \xi_{0}, \varphi_{0}\right)$ are obtained by fitting the experimental data in the angular region $|\varphi| \geq 100^{\circ}$ (where the NE emission is negligible) by the equilibrium multiplicity $[M(\varphi)]_{E}$, given by Eqs.(4),(8),(9). Then, the parameters $\left(C_{N E}, H_{0}, \Gamma_{+}, \Gamma_{-}, \chi_{0}\right)$ are obtained by fitting the experimental data in the complete angular region $|\varphi| \leq 180^{\circ}$ by the total multiplicity $[M(\varphi)]_{E}+[M(\varphi)]_{N E}$, given by Eqs.(4),(8)-(10), where the above determined values of the parameters $\left(C_{E}, \gamma, \zeta_{0}, \xi_{0}, \varphi_{0}\right)$ were inserted. Best $\chi^{2}$ values, estimated within $5 \%$, are listed in Table 1.

The in-plane differential multiplicities corresponding to the parameters listed in Table 1 are represented in Fig.1. The dashed and solid lines correspond to the equilibrium $M_{E}$ and total $M_{E}+M_{N E}$ multiplicities, respectively. The above semiclassical approach seems to be able to reproduce many of the observed features of the experimental in-plane angular correlations. The two angular bumps are associated to opposite polarizations of the fissioning PL fragment, the higher one with positive polarization and the lower one with negative polarization [3].

For each range of the total energy $E_{2 F}$, for larger $\left(A_{H} / A_{L}\right)$ the quantal deflection $\chi_{0}$ is smaller and consequently the two angular bumps merge 
Table 2: Estimates of the in-plane integrated $M_{E}$ and $M_{N E}$ multiplicities, the positive alignment $p_{+}$and the "formation-to-fast fission lifetime" $\tau_{0}$, in units $(\mathrm{fm} / \mathrm{c})$, for three ranges of the total kinetic energy of the two heaviest fragments $E_{2 F}$, in units $M e V$, and for three heavy-light mass ratios $\left(A_{H} / A_{L}\right)$.

\begin{tabular}{cccccc}
\hline \hline$E_{2 F}$ & $\left(A_{H} / A_{L}\right)$ & $M_{E}$ & $M_{N E}$ & $p_{+}$ & $\tau_{0}$ \\
\hline \multirow{3}{*}{$3200-3650$} & $2.6-4.6$ & 0.2 & 5.3 & 0.83 & 352 \\
& $1.6-2.6$ & 0.6 & 2.5 & 0.66 & 369 \\
& $1.0-1.6$ & 0.7 & 1.6 & 0.65 & 401 \\
\hline \multirow{3}{*}{$2850-3200$} & $2.6-4.6$ & 0.2 & 6.4 & 0.78 & 305 \\
& $1.6-2.6$ & 0.4 & 4.2 & 0.67 & 349 \\
& $1.0-1.6$ & 0.7 & 1.2 & 0.63 & 363 \\
\hline \multirow{2}{*}{$2500-2850$} & $2.6-4.6$ & 0.1 & 6.2 & 0.77 & 235 \\
& $1.6-2.6$ & 0.4 & 5.0 & 0.66 & 246 \\
& $1.0-1.6$ & 1.0 & 1.7 & 0.62 & 267 \\
\hline \hline
\end{tabular}

together (see Fig.1) whereas the parameter $\varphi_{0}$, which is the angular gap between $\vec{k}_{0}$ and $\vec{k}_{B}$ (corresponding to $\varphi=0^{\circ}$ ), is larger.

From Eqs.(9) and (10) one can obtain a rough estimate of the in-plane $\mathrm{E}$ and NE multiplicities integrated in the angular region $|\varphi| \leq 180^{\circ}$. The values of integrated multiplicities $M_{E}$ and $M_{N E}$ coming from the parameters $\left(C_{E}, C_{N E}, \Gamma_{+}, \Gamma_{-}, H_{0}\right)$ of Table 1 , estimated within $30 \%$, are listed in Table 2 .

We can define a positive alignment parameter $p_{+}$of the fissioning $\mathrm{PL}$ fragment, on a quantization axis perpendicular to the reaction plane [3], whose values, corresponding to the parameters $H_{0}$ given in Table 1 , estimated within 10\%, are listed in Table 2 .

From Eq.(11) with the values of $\chi_{0}$ given in Table 1 we can estimate the "formation-to-fast fission lifetime" $\tau_{0}$. We used $\ell_{0}=30 \hbar[2]$ and we calculated the moment of inertia of the fissioning nucleus $B(\equiv C+c)$ assuming a rigid rotation of two hard spheres, at contact [3]. The values of $\tau_{0}$, in units $(\mathrm{fm} / \mathrm{c})$, estimated within $15 \%$, for the three mass ratios $\left(A_{H} / A_{L}\right)$, are listed in Table 2.

The decrease of the lifetimes $\tau_{0}$ with increasing mass ratio $\left(A_{H} / A_{L}\right)$, for each range of the total energy $E_{2 F}$, is in clear agreement with results reported in Ref. [8]. 


\section{References}

[1] P. Russotto et al., Phys. Rev. C 91 (2015) 014610.

[2] E. De Filippo et al., Phys. Rev. C 71 (2005) 064604.

[3] A. Strazzeri and A. Italiano, Int. J. Mod. Phys. E 23(2014)1450081.

[4] A. Strazzeri, Nuovo Cimento A 52 (1979) 323.

[5] D.M. Brink and G.R. Satchler, Angular Momentum (Oxford University Press, Oxford, 1971).

[6] R.M. Green, Spherical Astronomy (Cambridge University Press, Cambridge, 1985).

[7] I. Halpern, Bull. Amer. Phys. Soc. II 5 (1960) 510.

[8] G. Casini et al., Phys. Rev. Lett. 71 (1993) 2567. 